\title{
SISTEMA DE GESTIÓN DE TRÁMITES Y LA APLICACIÓN DEL MODELO DE NEGOCIOS CANVAS
}

\author{
Ana Cecilia Díaz Rodríguez ${ }^{1}$, Ramón René Palacio Cinco $^{2}$, Martín Humberto Córdova Cárdenas ${ }^{3}$ \\ 'Unidad Navojoa,Instituto Tecnológico de Sonora anadiaz_712@hotmail.com 0000-0002-3710-1062 \\ 2 ramon.palacio@itson.edu.mx 0000-0002-4059-2149 \\ 3 martin.cordova@itson.edu.mx 0000-0001-5837-517X
}

\section{DOI: https://doi.org/10.46589/rdiasf.vi36.385}

Recibido 26 de junio 2021.

Aceptado 23 de septiembre 2021

Publicado 28 de octubre 2021

\section{Resumen}

En este artículo se tiene como propósito principal diseñar el modelo de negocios para la gestión de trámites de una oficina de gobierno. Esto se abordó debido a la necesidad de conocer cómo mejorar la gestión burocrática en cuanto al plazo de respuesta establecidopor la misma normativa para la resolución de los trámites, pues se ha rebasado dicho plazo,cabe mencionar que la mayoría de los trámites están sin concluir. Esto ha traído usuarios inconformes que necesitan dicha resolución para poder trabajar, por lo cual se han presentados demandas a la institución por no cumplir con el tiempo transcurrido. Para esto se realiza el análisis de la oficina basado en la metodología del Modelo de Negocios Canvasa través de la definición de sus nueve elementos clave, con lo cual los responsables de esta oficina puedan tener de una manera ágil y flexible una perspectiva holística de lo que conlleva el mejorar los tiempos de respuesta para los usuarios de esta oficina. Como resultados se obtiene la descripción de cada uno de los elementos que facilitan a los responsables la definición de objetivos medibles para la mejora de tiempos de respuesta a sus usuarios. Como trabajo futuro se propone diseñar iniciativas mediante la gestión estratégica y elementos que describen la propuesta de valor con el uso de mapeo de procesos.

Palabras clave: Modelo de negocios Canvas, Estructura de negocio, Gestión de trámites 


\section{Abstract}

The main purpose of this article is to design the business model for managing the procedures of a government office. This was addressed due to the need to know how to improve bureaucratic management in terms of the response period established by the same regulations for the resolution of procedures, since this period has been exceeded, it is worth mentioning that most of the procedures are unfinished. This has brought dissatisfied users who need this resolution to be able to work, for which demands have been presented to the institution for not complying with the time that has elapsed. For this, the analysis of the office is carried out based on the methodology of the Canvas Business Model through the definition of its nine key elements, with which those responsible for this office can have in an agile and flexible way a holistic perspective of what which entails improving response times for users of this office. As a result, a description of each of the elements is obtained that makes it easier for managers to define measurable objectives to improve response times to their users. As future work, it is proposed to design initiatives through strategic management and elements that describe the value proposition with the use of process mapping.

Keywords: Canvas business model, Business structure, Procedures management

\section{Introducción}

La Administración Pública Federal (APF), también se le denomina como Sistema de Administración del Gobierno Federal y cumple con las funciones asignadas por el poder ejecutivo federal, realiza las actividades designadas por el Presidente de la República, y tiene el respaldo directo en las Secretarías y Dependencias del Estado. La APF se encarga de satisfacer las necesidades, planes y proyectos de la comunidad a través de las instituciones con atribuciones propias del Estado (Secretaria de la Función Pública). Asimismo, en (Serra Rojas, 2020), se define el concepto de la APF como un conjunto de órganos que apoyan al Ejecutivo Federal en las prácticas administrativas, y se compone por la administración centralizada y administración paraestatal. La Administración Pública centralizada está compuesta por la Oficina de la Presidencia de la República, las Secretarías de Estado, la Consejería Jurídica del Ejecutivo Federal y los Órganos Reguladores Coordinados. La 
administración Pública paraestatal se integra de empresas de participación estatal; organizaciones nacionales de crédito; instituciones nacionales de seguros, de finanzas; y en organismos descentralizados, que son entidades creadas con personalidad jurídica y patrimonio propios.

Por decreto presidencial publicado en el diario oficial de la federación, Comisión Nacional del Agua (CONAGUA) nace el 16 de enero de 1989, como un órgano administrativo desconcentrado de la Secretaría de Agricultura y Recursos Hidráulicos (Arellano, 1989). LaComisión Nacional del Agua es heredada de la cultura hidráulica, que tiene la facultad de administrar, regular, controlar y salvaguardar las aguas nacionales y sus bienes públicos inherentes, con la responsabilidad del poder ejecutivo, legislativo, judicial y la comunidad en general, para alcanzar el uso sustentable del agua, prevenir riesgos originados de fenómenos meteorológicos y evaluar los efectos del cambio climático en el ciclo hidrológico. CONAGUA se divide operativamente en tres áreas: 1) Oficinas Centrales (la sede), dentro de sus gestiones ésta apoyar a las otras dos áreas, en la ejecución de sus labores para obtenerel uso sostenible del agua, implantar políticas y estrategias hidráulicas, proveer el presupuesto y vigilar su aplicación, constituir los programas de ayudar a los municipios en la provisión de los servicios de agua para promover su uso eficiente; 2) Organismos de Cuenca, su compromiso es administrar las trece regiones hidrológicas que hay en el país, determinando la disponibilidad de agua, lograr el uso sostenible del agua, preservar los acuíferos, solucionar conflictos relacionados con el agua, prevenir riesgos y promover la cultura del buen uso delagua, etcétera; y 3) Direcciones locales, tienen la tarea de aplicar las políticas, estrategias y programas de la CONAGUA en las entidades federativas que les corresponden (agua.org.mx, 2010).

Acorde a (Pérez Campos \& Priego Carrera, 2014), entre los procesos clave de CONAGUA está, planear, organizar, dirigir y controlar las acciones hidrológicas en la región por medio de la ejecución del Programa Hídrico Regional para la administración y preservación del uso sustentable del agua y sus bienes públicos inherentes, así como coordinar las acciones necesarias para la gestión, conservación y modernización de la infraestructura Hidroagrícola y la tecnificación de las zonas de riego de los distritos de riego, para optimizar el aprovechamiento de los recursos hidráulicos disponibles y 
económicos asignados.

Asimismo, entre sus funciones esta la elaboración de los proyectos para la gestión y funcionamiento técnico de los distritos de riego, elaboración de sus reglamentos, autorización de cuotas, organización y registro de los usuarios de los sistemas de riego, conservación, rehabilitación y modernización de obras para la operación de la infraestructura de riego y de recuperación de inversiones; realizar los proyectos para la transferencia de distritos a los usuarios, manejo del agua y preservación de suelos, elaboración y autorización de planes de riego y programas de conservación de obras, títulos de concesión, así como lo referente a la expedición de permisos únicos de siembra con derecho de agua; participar en acciones de promoción de créditos y otros mecanismos para el financiamiento de proyectos de equipamiento, rehabilitación y modernización de infraestructura Hidroagrícola y tecnificación del riego parcelario en los distritos; y coordinar la integración de informes referentes a los volúmenes de agua propuestos a utilizar por los distritos de riego, con la participación de los organismos de cuenca.

Por lo anterior, este trabajo tiene como objetivo diseñar el modelo de negocios para la gestión de trámites de una oficina de gobierno, mediante el Modelo de Negocios Canvas conla finalidad de mejorar su propuesta de valor actual.

\section{Planteamiento del problema}

Hoy en día, la sociedad tiene una opinión desfavorable de la administración pública, sin embargo, el desarrollo de una nación se basa en la confianza y participación ciudadana en los asuntos públicos. Los nuevos esquemas de gobernanza reclaman un rol ciudadano más activo y una gestión pública que rinda resultados y sea eficiente y abierta a la vez (SFP, 2012).

El gran reto que tienen hoy en día todas las organizaciones, sean públicas o privadas, es conseguir que el cliente o ciudadano en el caso de las administraciones se sienta satisfecho y que sus necesidades este cubierta, máxime si se habla de una sociedad de servicios basada enuna nueva dinámica social y económica donde diariamente se producen nuevos retos y cambios, desde la llegada de nuevas tecnologías hasta el impacto de nuevas normativas, globalización, clientes y usuarios con más opciones donde elegir incluso mejor 
informados y conocedores de sus derechos (Villa Casal, 2014).

Aun cuando una parte importante de las instancias productoras de bienes y proveedoras de servicios han aplicado estrategias de mejora y de búsqueda de ampliación de la satisfacción ciudadana, se mantienen vigentes pautas de organización y de provisión que otorgan un mayor peso a la reproducción de esquemas clientelares, al manejo selectivo de la oferta y la distribución de los bienes y servicios, al uso de criterios que privilegian y protegenlos intereses de los prestadores por encima de los usuarios o consumidores, y al mantenimiento de un marco regulatorio centrado en las instituciones y no en las demandas y necesidades ciudadanas (INAP, 2008).

Para enfocar objetivos dentro de la administración pública, para la mejora de un procesoo estructura de negocio, se propone el modelo de negocios como una herramienta para implementar estrategias, pues se considera como un instrumento para explotar oportunidades. Los modelos de negocios (Castillo, 2017) son estructuras cognitivas que brindan una teoría sobre cómo establecer límites para la empresa, cómo crear valor y cómo organizar su estructura interna y su dirección (Behnke \& Kibbel, 2017) .

En la práctica, el modelo de negocio se utiliza para analizar las empresas con el fin de obtener una visión más profunda de las actividades de la empresa (McGrath, 2010). La tarea principal del modelo de negocio es comercializar la innovación de manera que la empresa pueda capturar el mayor valor posible de ella (Zott et al., 2011). El modelo de negocios consiste en detallar por medio de un instrumento que es lo que se va a brindar, quienes van a ser los clientes, y de qué manera se va ofrecer, este instrumento permite analizar la misión, visión y objetivos del modelo de negocios, así como definir las fuentes de ingresos y costes que va a tener dicho modelo (Osterwalder \& Pigneur, 2009; Osterwalder \& Pigneur 2012). Dado lo anterior, planteamos la siguiente pregunta de investigación, ¿Cuáles son los elementos clave de la gestión de trámites para una oficina de gobierno que administra las aguas nacionales, en cuanto al plazo de respuesta establecido por medio de un sistema de gestión y seguimiento de trámites? 


\section{Método}

\section{Tipo de investigación}

El tipo de investigación de este trabajo tiene un enfoque cualitativo, pues se busca comprender cómo el modelo de negocio de la oficina de trámites se comporta frente a sus usuarios en cuanto al tiempo de respuesta del servicio otorgado. Para esto principalmente se utilizaron entrevistas y el análisis de los datos que registran los servicios o trámites prestados por la oficina a sus usuarios.

\section{Sujeto de estudio.}

El sujeto de estudio para esta investigación es la gestión y seguimiento de trámites que serealizan en el departamento de Administración del Agua de la institución en estudio como semuestra en la Tabla 1: en el año 2017 se registró la solicitud de 84 trámites de los cuales 33 se concluyeron; en el 2018, hubo 160 solicitudes de trámites y solo 56 tuvieron respuesta para concluir; se registraron 41 solicitudes de trámites en este año de los cuales no se ha tenido respuesta hasta la fecha. Dicho registro se encuentra en la base de daros que arrojo SIAA en las bitácoras en cuanto al número de trámites y tiempo de respuesta de los últimos tres años $(2017,2018$ y 2019) en el cual se consideraron los tramites que se realizan en la Ciudad de Navojoa, Sonora.

La Misión de la oficina es preservar las aguas nacionales y sus bienes públicos inherentes para su administración sustentable y garantizar la seguridad hídrica con la corresponsabilidad de los órdenes de gobierno y la sociedad en general. Su Visión, ser una institución de excelencia en la preservación, administración de las aguas nacionales y la seguridad hídrica de la población.

Por lo que podemos notar que el propósito de esta oficina en estudio es proporcionar la atención adecuada al usuario de aguas nacionales y bienes públicos inherentes, en forma rápida y eficiente para lograr su incorporación al régimen legal, mediante la emisión de títulos de concesión, asignación y permisos para el uso, explotación y aprovechamiento de los mismos. 
Tabla 1. Tipo de trámites y duración.

\begin{tabular}{|c|c|c|c|c|c|c|}
\hline AÑo & TPO DE TRAMITE & CODOKO & $\begin{array}{l}\text { TRAMTES } \\
\text { CONCLUDOS } \\
\text { EN TEMPO }\end{array}$ & $\begin{array}{l}\text { TRAMITES } \\
\text { CONCLUIDOS } \\
\text { EN } \\
\text { DESTIEMPO }\end{array}$ & $\begin{array}{l}\text { TRAMITES } \\
\text { SW } \\
\text { CONCLUIR }\end{array}$ & $\begin{array}{l}\text { PRCNEDIO DE } \\
\text { DURACION DE } \\
\text { TRANATES } \\
\text { CONCLUIDOS }\end{array}$ \\
\hline $201 ?$ & 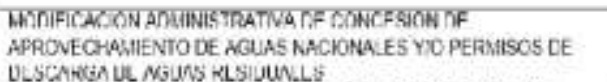 & CONACHAG-11-01] & 0 & 0 & 4 & 9 \\
\hline 2017 & 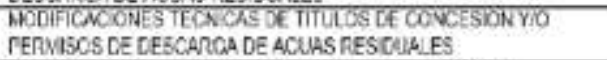 & COAACURAT-0र & 0 & 3) & B & Tarovocas \\
\hline $201 ?$ & $\begin{array}{l}\text { AUTORIZRCION PARA LA TRARSMISICN CE TITULOSY SU } \\
\text { REGISTRO }\end{array}$ & 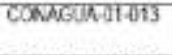 & 0 & 7 & 23 & $11730 \mathrm{cas}$ \\
\hline 2017 & 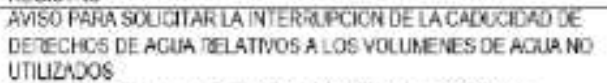 & 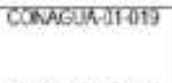 & 0 & 7 & 7 & 2285 cas \\
\hline 2017 & 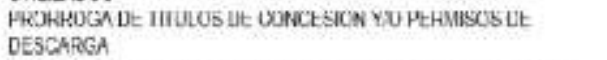 & CONABSUA-01-021 & 0 & 18 & 18 & 27937 cass \\
\hline 2013 & 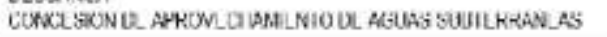 & COWACUAS-11-0D4 & 0 & c & 4 & 0 \\
\hline 2013 & 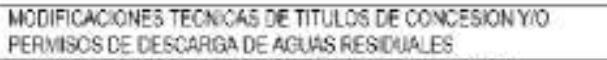 & COWWEA U1 012 & 0 & e & 23 & $\overline{9}$ \\
\hline 2018 & $\begin{array}{l}\text { NUIORILCIONPMMLATRMSMISICW LE TIULOSY SU } \\
\text { RESISTZO }\end{array}$ & 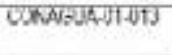 & iv & $T$ & $2 \pi$ & TWW Cias \\
\hline 208 & 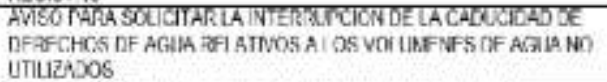 & 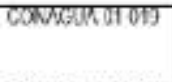 & (1) & 15 & 75 & $2340 \mathrm{cas}$ \\
\hline कात & 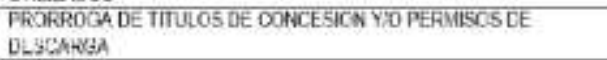 & 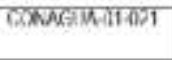 & $\pi$ & का & $2 \pi$ & Tिक 15 Gas \\
\hline 2018 & Dसिमकी० & & 0 & 0 & 22 & 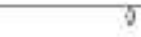 \\
\hline 2019 & 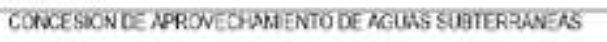 & 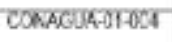 & 0 & 6 & t & 8 \\
\hline 2019 & $\begin{array}{l}\text { WODIFICACONES TECKCAS DE TITULOS DE COVCESIONYO } \\
\text { PERUASOS DE DESCARON DE NGUNS RESCUNLES }\end{array}$ & conasurate12 & 0 & e & 4 & 0 \\
\hline 2019 & $\begin{array}{l}\text { MUTORIZACIDN PARA LA TRRESMISICN DE TIULLOS Y SU } \\
\text { HLGISINO }\end{array}$ & COKACUA- $01-013$ & 0 & e & 13 & 0 \\
\hline 2019 & 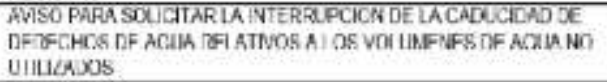 & CONAGUAF-OT-OT & 0 & 0 & 2 & $\overline{0}$ \\
\hline$\% 019$ & $\begin{array}{l}\text { PROFROGA DC TITULOS DC CONCESICN YYO PCFMISOS DC } \\
\text { DESCARGA }\end{array}$ & 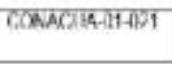 & 0 & 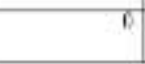 & 21 & $\overline{9}$ \\
\hline
\end{tabular}

Fuente: Software SIAA.

La muestra de datos se recolectó mediante el Sistema Integral de Administración del Agua (SIAA), este sistema es exclusivo de la Comisión Nacional del Agua. Esta información se procesó en Excel, con datos desde el 2017 hasta 2019, obtenidos en el SIAA, el cual está dividido 6 columnas: 1) Año en el que se realizó el trámite; 2) Nombre del trámite; 3) Códigodel trámite asignado por la dependencia; 4) Fecha de inicio del trámite; 5) Fecha en la que seconcluyó el trámite; y 6) Tiempo de respuesta de conclusión del trámite. 


\section{Materiales.}

Se utilizó el Reglamento de Plazos de respuesta y cuotas por derechos de expedición y registro en el cual se describe el nombre del trámite, así como el número de días que se le otorga a cada trámite para plazo de respuesta y la cuota que se cobra para realizarlo. También se accedió al Sistema de Integración de Administración del Agua (SIAA) en el apartado de Administración del Agua para obtener la base de datos de los trámites realizados en la Ciudad de Navojoa, Sonora.

También se utilizó el Modelo de Negocios Canvas, este ayuda a identificar los aspectos más importantes del negocio planificado, este modelo está basado en la experiencia práctica de emprendedores de todo el mundo (Tokarski, Tokarski, \& Wójcik, 2017). El Modelo CANVAS es una plantilla donde se visualizan y describen los componentes para el desarrollo de la gestión estratégica de un modelo de negocio, y crear una propuesta que le genere valora la empresa (Casañas Villar \& Barbosa Iglesias, 2016). Según (Osterwalder \& Pigneur, 2012) este modelo de negocio consiste en nueve elementos básicos (ver Figura 1) que encajan en la lógica del proceso de fabricación de la empresa, estos nueve elementos tocan cuatro áreas principales de la actividad comercial, los clientes, las ofertas, infraestructura y posiciónfinanciera adecuada. El modelo de negocio está en una forma de bosquejo de la estrategia a ser implementada dentro de las estructuras, procesos y sistemas de una organización. 
Figura 1. Los 9 bloques para analizar el Modelo CANVAS.

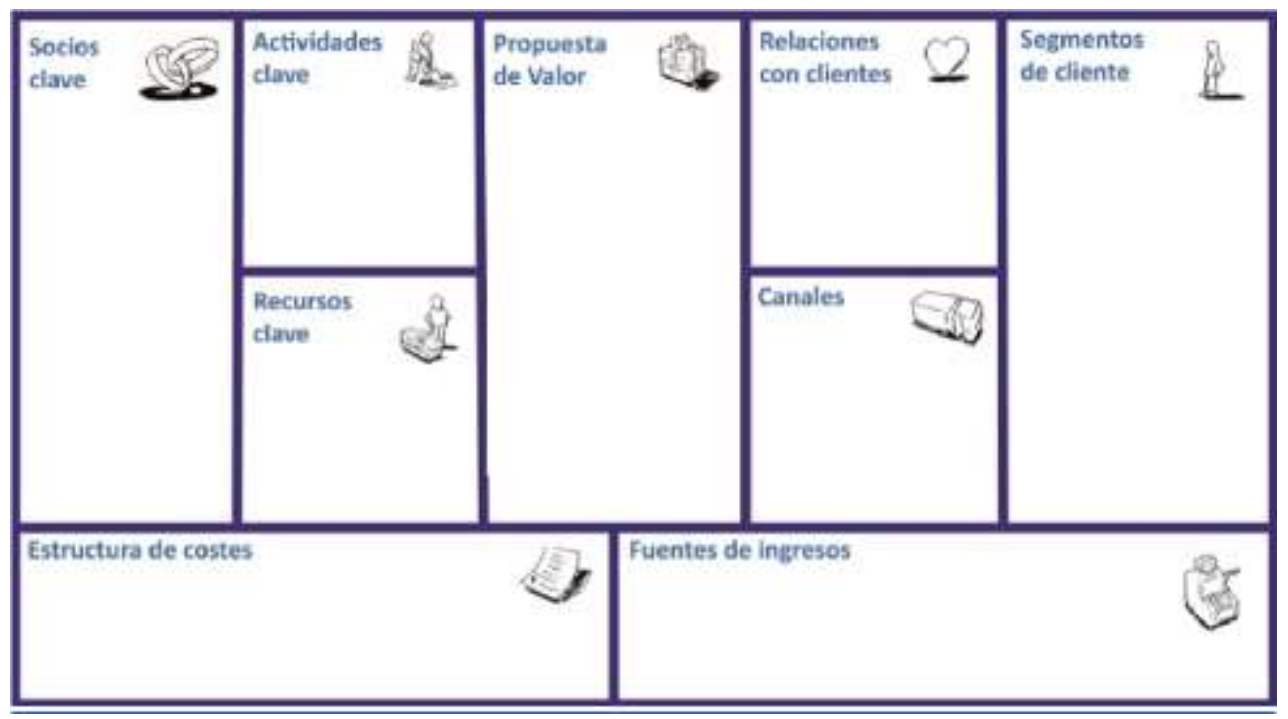

Fuente: (Osterwalder, A., 2014).

\section{Procedimiento.}

Este trabajo de investigación se realizó en 4 pasos, los cuales se describen a continuación:

1. Entrevista inicial. Se realizó una entrevista al responsable de la ventanilla única de la gestión de trámites con el fin de conocer los diferentes procesos que constan desde la entrada que se solicita un servicio hasta que se obtiene la respuesta final del trámite.

2. Análisis de datos. Se analizó la base de datos del sistema SIAA para obtener los tipos y número de trámites que se realizan durante un período, y su tiempo de respuesta. Entre los hallazgos importantes se pudo observar que la resolución de los trámites ha rebasado dicho plazo alcanzando hasta 810 días de retraso, mientras que la normativa señala un plazo de hasta 60 días hábiles, cabe mencionar que la mayoría de los trámites están sin concluir. Esto ha traído usuarios inconformes que necesitan dicha resolución para poder trabajar, por lo cual se han presentados demandas a la institución por no cumplir con el tiempo transcurrido.

3. Segmentación de clientes. se realizó una segmentación de los usuarios que utilizan la ventanilla única para los trámites con el uso de formatos de diseño del segmento de 
clientes propuesto por (Mouriño, 2018) que fueron llenados por el personal responsable.

4. Formatos "value proposition". Con la información obtenida en la segmentación y la información recopilada de la base de datos y la entrevista al personal responsable, se elaboraron las propuestas de valor y el mapa de empatía (Sánchez Vásquez et al., 2016) que ayudaron a la elaboración del Modelo de Negocio Canvas.

\section{Resultados}

Con el fin de establecer el conocimiento que la Gestión de Trámites requiere de las diversas áreas que la conforman, se describen los elementos clave que resultaron en el Modelo de Negocio Canvas. A continuación, se describen cada uno de ellos:

1) Segmentación de clientes: Para realizar la segmentación de clientes del departamento, se extrajo del Manual Authentic Leaders, Emprendimiento basado en innovación e impacto social, el formato "Diseño de segmento de clientes" con la información extraída del programa SIAA, la cual arrojo la siguiente información: los usuarios de CONAGUA del Distrito de Riego 038, Rio Mayo está compuesto por personas físicas y morales entre edades de 27 hasta los 95 años, con una edad promedio de 57 años; se tiene un porcentaje de usuarios como personas morales del $43 \%$ y un $57 \%$ de personas físicas. Sin embargo, en base a la experiencia del personal de atención al cliente se realizó el Mapa de Empatía por Macías (2011) que indica que los usuarios de CONAGUA están interesados en que se les resuelvan los tramites, ya que buscan estar bien con las leyes nacionales para así poder seguir aprovechando de los bienes y aguas nacionales, en el caso de los tramites de AUTORIZACIÓN PARA LA TRANSMISIÓN DE TÍTULOS Y SU REGISTRO no pueden perforar si no tienen la resolución del volumen o derecho adquirido, así mismo en los tramites de

MODIFICACIONES TÉCNICAS DE TÍTULOS DE CONCESIÓN Y/O PERMISOS DE DESCARGA DE AGUAS RESIDUALES por reposición o 
relocalización de volumen, los usuarios no pueden extraer el volumen. Existen multas entre los 5,000 a 500,000 salarios mínimos dependiendo de la gravedad respecto a la ley de aguas nacionales. No obstante, después de pasar el tiempo de respuesta establecido por CONAGUA, el usuario tiene derecho a poder denunciar a la misma por no haber obtenido una resolución en ese plazo transcurrido.

2) Propuesta de Valor: La propuesta de valor para este Modelo de Negocios es reducir el tiempo de respuesta en los trámites: CONAGUA-01-001, CONAGUA-01-004, CONAGUA-01-010, CONAGUA-01-012, CONAGUA-01-013, CONAGUA01019, CONAGUA-01-021, según el plazo especificado en la normativa de CONAGUA.

3) Aliados Clave: Se cuenta con el apoyo de personal a cargo de diversos departamentos en la Ciudad de Hermosillo, los cuales le dan seguimiento a cada trámite para la verificación y autorización correcta para el cumplimiento de las leyes, así mismo se cuenta con un Área Jurídica encargada de sancionar cualquier delito que se incurra. Otro aliado clave en este proceso es la Secretaria de Hacienda y Crédito Público, quien otorga el presupuesto a este departamento.

4) Actividades Clave: Entre las actividades clave en este proceso están las notificaciones a los usuarios por alguna anomalía o requisición, realizar gestión de permisos, otorgar concesiones, actividades de mensajería, así como también visitas de supervisión y así verificar que se estén cumpliendo las normas. Cuando un usuario hace la solicitud de algún trámite, el encargado de Ventanilla recibe la documentación requerida para integrar el expediente, posteriormente envía la documentación a la ciudad de Hermosillo al departamento de Dictaminación, donde el personal a cargo analiza dicho expediente, de ahí se pasa al Área Técnica para verificar el aprovechamiento del acuífero donde se está solicitando el trámite, después se regresa a Dictaminación para analizar la documentación verificada por el Área Técnica, ya que todo este revisado, analizado y autorizado, el departamento de Titulación, elabora la resolución del título, son quienes aprueban o no el seguimiento del trámite, una vez autorizado el director de CONAGUA firma la documentación, y se pasa al departamento de REPDA 
para tomar el Registro Nacional del aprovechamiento. Por último, la documentación regresa al departamento de Ventanilla Única para notificar y entregar la resolución del trámite al Usuario.

5) Recursos Clave: Para que todo el proceso se lleve a cabo se necesitan una serie de recursos como equipo de cómputo, teléfono, conmutador, vehículos oficiales, GPS (Para visitas de inspección), impresora, escáner, sellos, artículos de papelería, internet, y sobre todo el Recurso Humano, todo esto para facilitar y tomar las acciones pertinentes de cada tramite.

6) Relación con los clientes: El personal a cargo de relacionarse y atender al usuario lo puede hacer de manera presencial en su domicilio, en las oficinas, por media atención telefónica, incluso por correo electrónico, para atender cualquier duda por parte del usuario, hacerle llegar cualquier notificación, o cualquier consulta respecto al trámite.

7) Canales: El usuario es la persona interesada en que su trámite se autorice y se realice de la mejor manera, es por ello que siempre debe de estar bien informado, para esto puede obtener información por medio de folletos y un portal en internet, así como también de manera telefónica o personalmente en las oficinas para comunicarse directamente con las personas capacitadas en ofrecer tal información.

8) Estructura de costes: Todo proceso tiene un costo, en este caso se toma en cuenta la nómica del personal que se necesita para cada fase en la gestión de los tramites, así como también el combustible para realizar notificaciones a los usuarios, y sobre todos gastos administrativos, como la luz, papelería, limpieza, etcétera.

9) Vías de Ingresos: Esta dependencia es una institución gubernamental que no cuenta con sus propios recursos, sin embargo, recibe cuotas de derechos de los usuarios, así como también presupuesto del gobierno para cubrir sus gastos.

Este Modelo de Negocio infiere un mejor planteamiento de objetivos por medio de la identificación de los distintos elementos del modelo en la propuesta de valor, generando objetivos mínimos viables para los responsables. También con este modelo se obtiene un panorama completo de los elementos que rodean a los diferentes servicios de esta oficina, 
con lo cual se pueden establecer iniciativas para establecer objetivos que en este caso apoyen a la reducción del tiempo de respuesta para los usuarios.

\section{Discusión}

En este artículo se diseña un modelo de negocio para la gestión de trámites en una oficinade gobierno utilizando la metodología del Modelo de Negocio Canvas, con la finalidad de que esta oficina pueda visualizar su propuesta de valor actual para reducir su riesgo en cuantoa los tiempos de respuesta a los usuarios, detectando oportunamente las áreas de oportunidad, debido a que con la información resultante los responsables o directivos de estos trámites puedan plantear estrategias y acciones coherentes a su misión y visión.

Esta oficina de gobierno no dispone de una plantilla Canvas, aun cuando los responsables de tomar decisiones requieren información disponible de su situación actual. Para esto el Modelo de Negocio Canvas permite que esta información sea generada de manera rápida y flexible, esto en apoyo a la alta dirección pues requieren diseñar y controlar no solo puntos clave para satisfacer a sus usuarios actuales, sino también incluir aspectos que permitan la buena marcha y desarrollo del modelo de negocio (Castillo, 2017). Es aquí donde se estableció la necesidad aplicar este modelo, ya que dicho modelo proporciona iniciativas y objetivos enfocados a medir y controlar los aspectos relevantes de esta oficina.

El Modelo de Negocios Canvas está limitado en ciertos aspectos que se deben considerar, entre ellas es el nivel de abstracción (Osterwalder \& Pigneur, 2009), lo cual dificulta la implementación de estrategias, al no proporcionar indicadores, ni relaciones que tienen los elementos clave involucrados en el modelo, por lo que se debe considerar el identificar las fortalezas y debilidades de cada proceso del modelo de negocio, para posteriormente crear iniciativas que ayudan a reducir las debilidades y consolidar las fortalezas.

\section{Conclusiones}

En este artículo se abordó la necesidad de conocer como diseñar los elementos clave del modelo de negocios para la gestión burocrática en cuanto al plazo de respuesta establecido 
por la normativa. Entre los hallazgos importantes se pudo observar que la resolución de los trámites ha rebasado dicho plazo alcanzando. Esto ha traído usuarios inconformes que necesitan dicha resolución para poder trabajar, por lo cual se han presentados demandas a la institución por no cumplir con el tiempo transcurrido. Por otro lado, se han presentado multas a los usuarios que incurren en la Ley de Aguas Nacionales, todo esto por los retrasos ocasionados en la conclusión de trámites.

Como una limitante de este trabajo, es que no se pudo tener relación directa para entrevistar a los usuarios, ya que en el tiempo que se realizó la investigación se presentaron pocas solicitudes de trámites, para esto se utilizó la historia de la base de datos del Sistema de Integración de Administración del Agua (SIAA), del cual se recolecto información referente al tipo de trámite que solicita cada usuario, así como también si fue concluido y encuanto tiempo, con información de los años 2017, 2018 y 2019.

La contribución de este trabajo es concretar el modelo de negocios de la institución en estudio, pues se identificaron los nueve componentes del CANVAS para crear la propuesta de valor de esta investigación. Es por ello, que para las empresarios o directivos que busquen ser los primeros en el mercado, que tengan una organización a su cargo y deseen agregarle valor o crear un nuevo negocio es recomendable analizar los nueve bloques del Modelo de Negocio CANVAS, el cual es de gran utilidad y se pueden identificar de manera clara y simple, además de que permite adaptarse a las nuevas propuestas del modelo de negocios quese desea obtener.

\section{Referencias bibliográficas}

agua.org.mx. (2010). Comisión Nacional de Agua (CONAGUA).

https://agua.org.mx/biblioteca/comision-nacional-de-agua-conagua/

Arellano, M. (1989). DECRETO por el que se crea hl Comisión Nacional del Agua. Diario oficial de la federación.

https://www.gob.mx/cms/uploads/attachment/file/110378/16_ENERO_1989_DEC 


\section{RETO_DE_CREACI_N CNA_COMO_ORGANO_ADMINISTRATIVO_DESC}

\section{ONCENTRADO_DE_SARH.pdf}

Behnke, I., \& Kibbel, F. (2017). The Business Model Innovation Process in High-Tech Start-ups. Business and Economics, 37(4), 1019-1042.

Castillo, S. (2017). Aplicación de Teoría C- K y modelo de negocios Canvas para asistir el diseño de nuevos servicios. Coloquio de investigación multidisciplinaria, Orizaba, Mex.

INAP. (2008). Mejora de la función pública. Revista de administración púbica, XLIII(3). https://inap.mx/wp-content/uploads/2020/09/INAP-RAP_117-2008.pdf

McGrath, R. (2010). Business Models: A Discovery Driven Approach. Long Range Planning, 43, 247-261.

Mouriño, J. (2018). Authentic Leaders: Emprendimiento basado en innovación e impacto. iLab.

Osterwalder, A., \& Pigneur, Y. (2009). Obstáculos de la innovación en los modelos de negocios. In Business Model Generation.

Osterwalder , A., \& Pigneur , Y. (2012). Business Model Generation (Hoboken, Ed.). John Wiley \& Sons, Inc.

Pérez Campos, M., \& Priego Carrera, M. (2014). MANUAL DE INTEGRACIÓN, ESTRUCTURA ORGÁNICA Y FUNCIONAMIENTO. CONAGUA. http://www.conagua.gob.mx/CONAGUA07/Contenido/Documentos/Manual de Integracion,EOyF.pdf

Sánchez Vásquez, J. M., Vélez Elorza, M. a. L., \& Pedro, A. j. P. n. (2016). Balanced scorecard para emprendedores: desde el Modelo Canvas al cuadro de mando. Revista Facultad de Ciencias Económicas: Investigación y Reflexión, 24(1).

Serra Rojas, A. (2020). Administración Pública Federal. Enciclopedia Jurídica Online. https://mexico.leyderecho.org/administracion-publica-federal/

SFP. (2012). Programa especial de Mejora de la Gestión en la Administración Pública 
Federal. SFP. http://2006

2012.funcionpublica.gob.mx/images/doctos/PROGRAMAS/pmg/manual_de_opera cion_pmg_2012.pdf

Villa Casal, J. P. (2014). Manual de atención a clientes y usuarios. BRESCA.

Zott, C., Amit, R., \& Massa, L. (2011). The Business Model: Recent Developments and Future Research. Journal of Management, 37(4), 1019-1042.

Cómo citar:

Díaz Rodríguez , A. ., Palacio Cinco, R. ., \& Córdova Cárdenas, M. . (2021). Sistema de gestión de trámites y la aplicación del modelo de negocios canvas. Revista De Investigación Académica Sin Frontera: División De Ciencias Económicas Y Sociales, (36), 1-15. Recuperado a partir de https://revistainvestigacionacademicasinfrontera.unison.mx/index.php/RDIASF/article/view/385

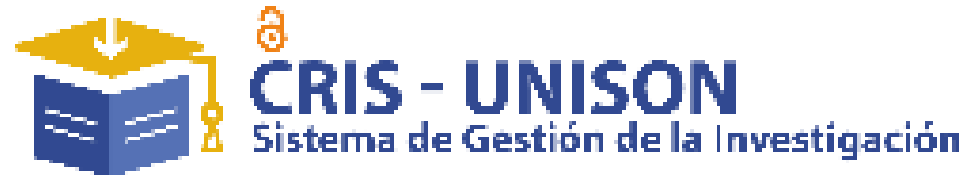
- Dialnet
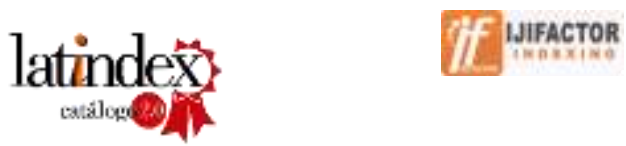

REDIB

CiteFactor

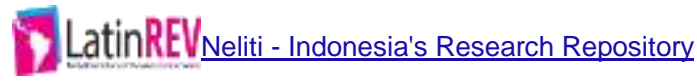
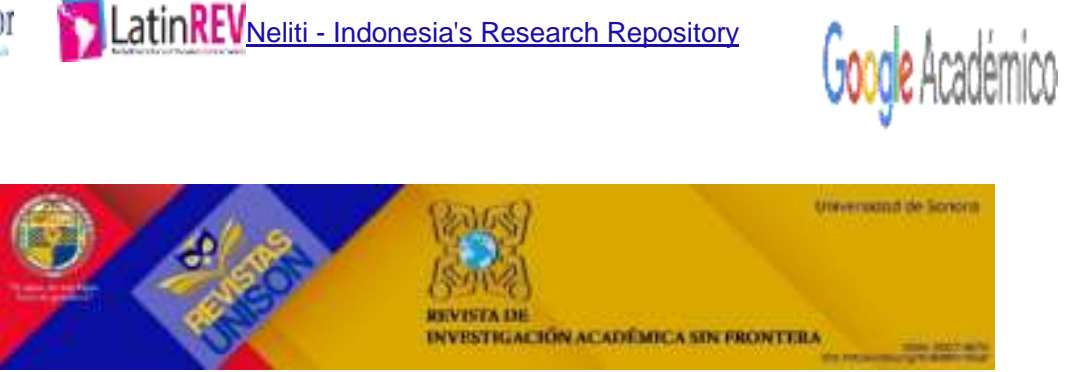